\title{
FIRST NUCLEAR-ASTROPHYSICS EXPERIMENTS WITH HIGH-INTENSITY NEUTRONS FROM THE LIQUID-LITHIUM TARGET LiLiT
}

\author{
M. Paul ${ }^{1}$ and M. Tessler
}

Racah Institute of Physics, Hebrew University

Jerusalem, Israel 91904

E-mail:paul@vms.huji.ac.il

G. Feinberg, S. Halfon, A. Arenshtam, O. Aviv, D. Berkovits, Y. Eisen, I. Eliyahu, G. Haquin, N. Hazenshprung, D. Kijel, A. Kreisel, I. Mardor, G. Shimel, A. Shor, I. Silverman, A. Sonn, L. Weissman and Z. Yungrais

Soreq Nuclear Research Center

Yavne, Israel 81800

\begin{abstract}
A high-intensity neutron source based on a Liquid-Lithium Target (LiLiT) and the ${ }^{7} \operatorname{Li}(p, n)$ reaction was developed at SARAF (Soreq Applied Research Accelerator Facility, Israel). The setup is used for nuclear-astrophysics experiments owing to the quasi-Maxwellian shape of the neutron energy distribution at stellar thermal energies $(k T \sim 30 \mathrm{keV})$. The LiLiT device consists of a forced-flown $(>2 \mathrm{~m} / \mathrm{s})$ film of liquid lithium $\left(\sim 200^{\circ} \mathrm{C}\right)$ whose free surface is bombarded by a proton beam. The lithium film acts both as the neutron-producing target and as a power beam dump. The setup was commissioned with a $1.2 \mathrm{~mA}$ proton beam at $1.91 \mathrm{MeV}$, producing a neutron yield (peaked at $\sim 28 \mathrm{keV}$ ) of $\sim 3 \times 10^{10} \mathrm{n} / \mathrm{s}$, more than one order of magnitude larger than conventional ${ }^{7} \mathrm{Li}(p, n)$-based neutron sources. The target dissipates a peak power areal density of $2.5 \mathrm{~kW} / \mathrm{cm}^{2}$ and a peak power volume density of $500 \mathrm{~kW} / \mathrm{cm}^{3}$ with no significant temperature or vacuum pressure elevation in the target chamber. We present preliminary results of first activation measurements on $\mathrm{Zr}$ and Ce stable isotopes performed with the SARAF-LiLiT setup, using $\mathrm{Au}$ as neutron monitor and of the determination of their Maxwellian-averaged neutron capture cross section.
\end{abstract}

XIII Nuclei in the Cosmos

7-11 July, 2014

Debrecen, Hungary

1

Speaker 


\section{Introduction}

The advent of high-intensity particle accelerators, generally based on radio-frequency (RF) linear structures, opens new avenues in different branches of nuclear physics and nuclear astrophysics. However, the associated high beam powers demand developing targets capable of dissipating powers of kilowatts without deterioration. The issue is particularly severe for the ${ }^{7} \mathrm{Li}(p, n)^{7} \mathrm{Be}$ reaction which has been widely used for the production of so-called quasi-Maxwellian $(k \mathrm{~T} \sim 30 \mathrm{keV})$ neutrons and the study of neutron-capture reactions of importance to the astrophysical s-process [1]. Conventional experimental systems (based on a Van de Graaff accelerator and solid Li or LiF targets) are limited to proton intensities below $100 \mu \mathrm{A}$ by the poor thermal properties of the target and neutron intensities of $\sim 10^{9} \mathrm{n} / \mathrm{s}$.

We have developed and commissioned a new metallic Liquid-Lithium Target (LiLiT) based on a Li film flowing at a speed of $2-5 \mathrm{~m} / \mathrm{s}$ and capable of sustaining up to $\sim 6 \mathrm{~kW}$ of proton beam power (1.9 MeV, $3 \mathrm{~mA})$, producing a neutron beam intensity of $\sim 2 \times$ $10^{10} \mathrm{n} / \mathrm{s} / \mathrm{mA}$. The target is currently used with the Soreq Applied Research Accelerator Facility (SARAF) high-intensity accelerator. We describe in the following sections the relevant properties of the SARAF accelerator, the LiLiT apparatus (see [2,3] for details) and first experiments aimed at studying neutron capture reactions of interest to nuclear astrophysics.

\section{The SARAF accelerator}

SARAF [4] is based on a superconducting linear accelerator for light ions $(m / q \leq 2)$, specially designed for high-intensity (up to 2-4 mA) beams. Phase I of SARAF (fig. 1) consists of an Electron Cyclotron Resonance Ion Source (ECRIS), a Low Energy Beam Transport (LEBT) section, a 4-rod Radio Frequency Quadrupole (RFQ) injector, a Medium Energy Beam Transport (MEBT) section and a Prototype Superconducting Module (PSM).

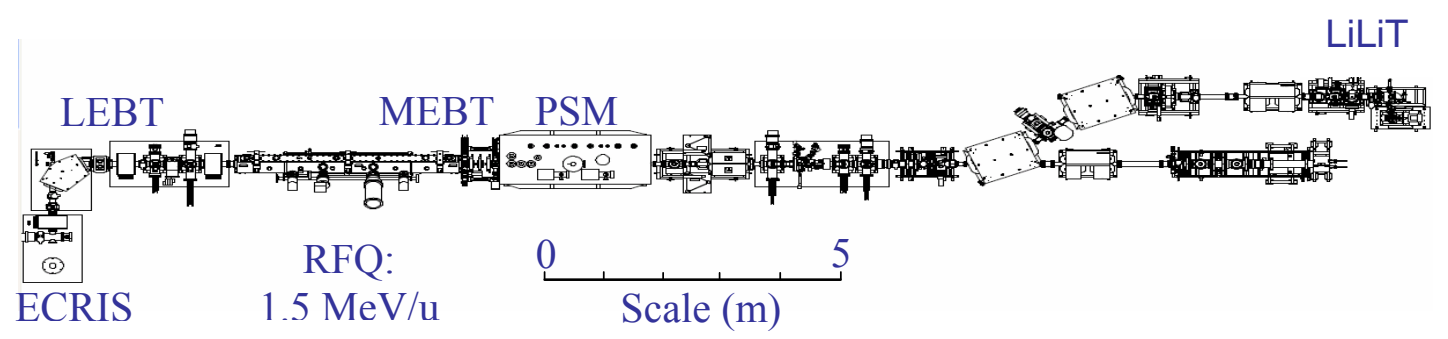

Figure 1: Schematic diagram of the SARAF Phase I high-intensity superconducting linear accelerator and the LiLiT beam line. See text for component acronyms. 
Currently under Phase I, continuous-wave (176 MHz) proton beams are accelerated by the RFQ and the PSM in the energy range 1.5-4 MeV at full intensity specifications [4]. Deuteron beams up to $\sim 5.5 \mathrm{MeV}$ are available at limited duty factor. Phase II, under design, is planned for a final energy of $40 \mathrm{MeV}$ at full beam intensity. A beam line dedicated to the LiLiT setup is available for experiments in the present configuration; a target hall is under planning and will be soon available for experiments.

\section{The Liquid-Lithium Target (LiLiT)}

The LiLiT assembly ([2], fig. 2) consists of a film of liquid lithium (at $\sim 200^{\circ} \mathrm{C}$, above the lithium melting temperature of $180.5^{\circ} \mathrm{C}$ ) flowing at high velocity onto a thin
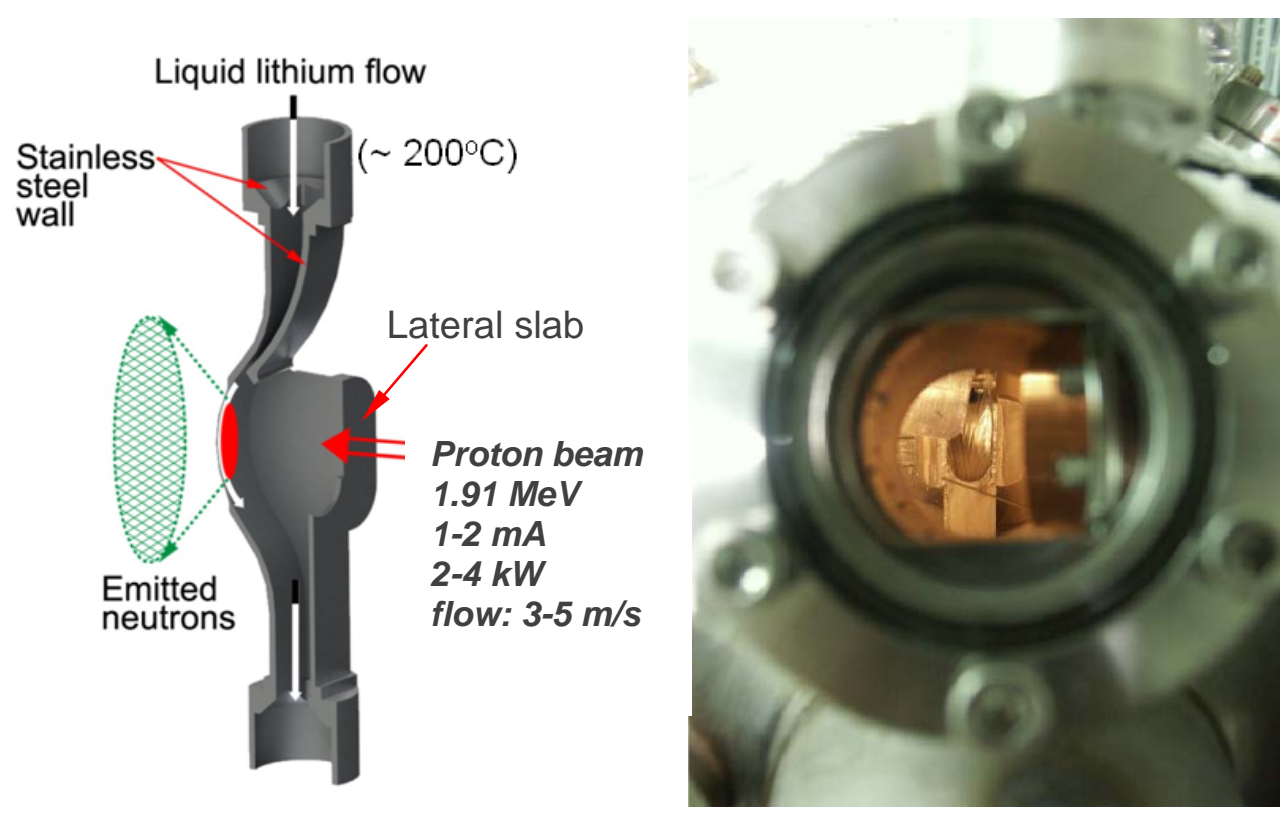

Figure 2: (left) Schematic isometric cross section diagram of the Liquid-Lithium Target (LiLiT). See [2,3] for details; (right) view of the free-surface liquid lithium flow photographed from the target chamber viewport.

stainless-steel wall with concave curvature towards the proton beam. A rectangularshaped nozzle just before the curved wall determines the film width and thickness (18 $\mathrm{mm}$ and $1.5 \mathrm{~mm}$, respectively). The target is bombarded by a high-intensity proton beam (1-2 mA) impinging directly (windowless) on the Li-vacuum interface at an energy $(1.91 \mathrm{MeV})$ above and close to the ${ }^{7} \mathrm{Li}(p, n)$ reaction threshold $(1.8804 \mathrm{MeV})$. 
The first few $\mu$ m's at the surface of the liquid-lithium film serve thus as a neutronproducing thick target (down to neutron threshold) and the deeper layers as a beam dump from which the power is transported by the flow to a heat exchanger. The setup takes into advantage the exceptionally high specific heat capacity of liquid lithium $\left(C_{p}=\right.$ $4350 \mathrm{~J} / \mathrm{kg} / \mathrm{K}$, as high as that of water) and its extremely low vapor pressure $\left(7 \times 10^{-9}\right.$ mbar at $220^{\circ} \mathrm{C}$ ). The flow velocity is set so that the steady-state local temperature in the beam spot area does not exceed a limit value determined by an allowed evaporation rate. The target dissipates in these conditions a peak power areal density of $2.5 \mathrm{~kW} / \mathrm{cm}^{2}$ and a peak power volume density of $500 \mathrm{~kW} / \mathrm{cm}^{3}$ with no major vacuum or temperature degradation in the target chamber. The temperature of lateral slabs on both sides of the proton beam (see fig. 2) is monitored by thermocouple gauges and allows one to steer the beam to the center of the lithium flow in the horizontal direction.
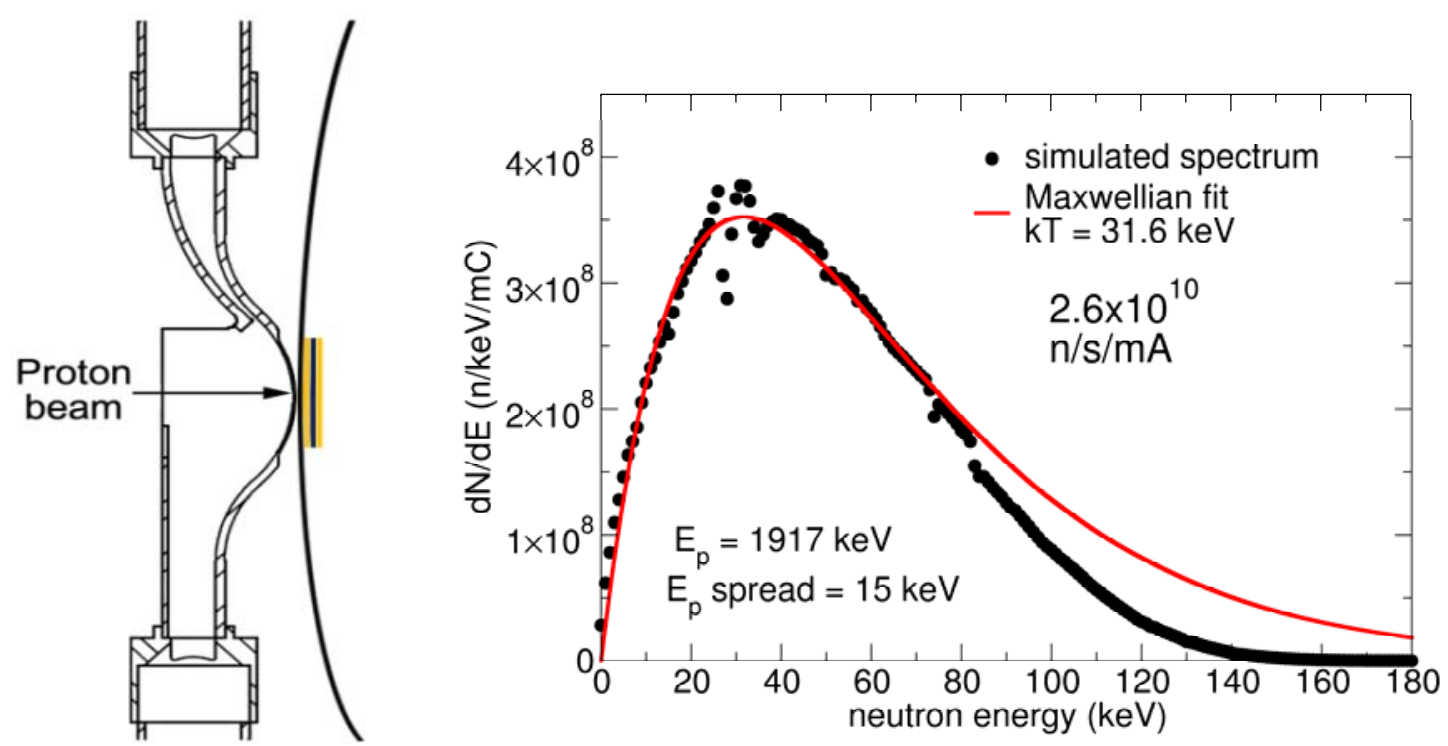

Figure 3: (left) Schematic diagram of the LiLiT and activation target setup. The activated targets (diameter $25 \mathrm{~mm}$ ) are positioned in the neutron cone in the center of a thin ( $0.5 \mathrm{~mm}$ stainless) wall of opposite curvature to the lithium film which separates the LiLiT chamber from an experimental scondary target chamber. The investigated target (dark blue) is sandwiched between two gold foils (orange) of same diameter serving as monitors of the neutron fluence; (right) neutron energy spectrum seen by the activation targets, calculated by a detailed simulation of the experimental system. The narrow dip at $\mathrm{E}_{n} \sim 26 \mathrm{keV}$ (a strong resonance in the system ${ }^{56} \mathrm{Fe}+n$ ) is due to interaction of outgoing neutrons in the stainless steel vacuum wall. The dashed line shows the fit of a Maxwell-Boltzmann flux of neutrons $v \cdot d n_{M-B} / d \mathrm{E}_{n} \alpha \mathrm{E}_{n} \exp \left(-\mathrm{E}_{n} / k \mathrm{~T}\right)$ for $k \mathrm{~T}=31.6 \mathrm{keV}$ to the spectrum seen by the targets. 


\section{Neutron activation measurements}

Activation targets (fig. 3 ) are positioned behind a curved wall ( $0.5 \mathrm{~mm}$ stainless steel) in a secondary vacuum chamber downstream of the LiLiT chamber, which acts also as a safety beam dump in case of accidental failure of the lithium flow; the curved wall (of curvature opposed to that of the lithium duct) allows the targets to be positioned close (6 $\pm 1 \mathrm{~mm}$ ) to the lithium surface in the outgoing neutron cone. The neutron intensity incident on the activation targets in these conditions is $\sim 3 \times 10^{10} \mathrm{n} / \mathrm{s}$ [3], forward-
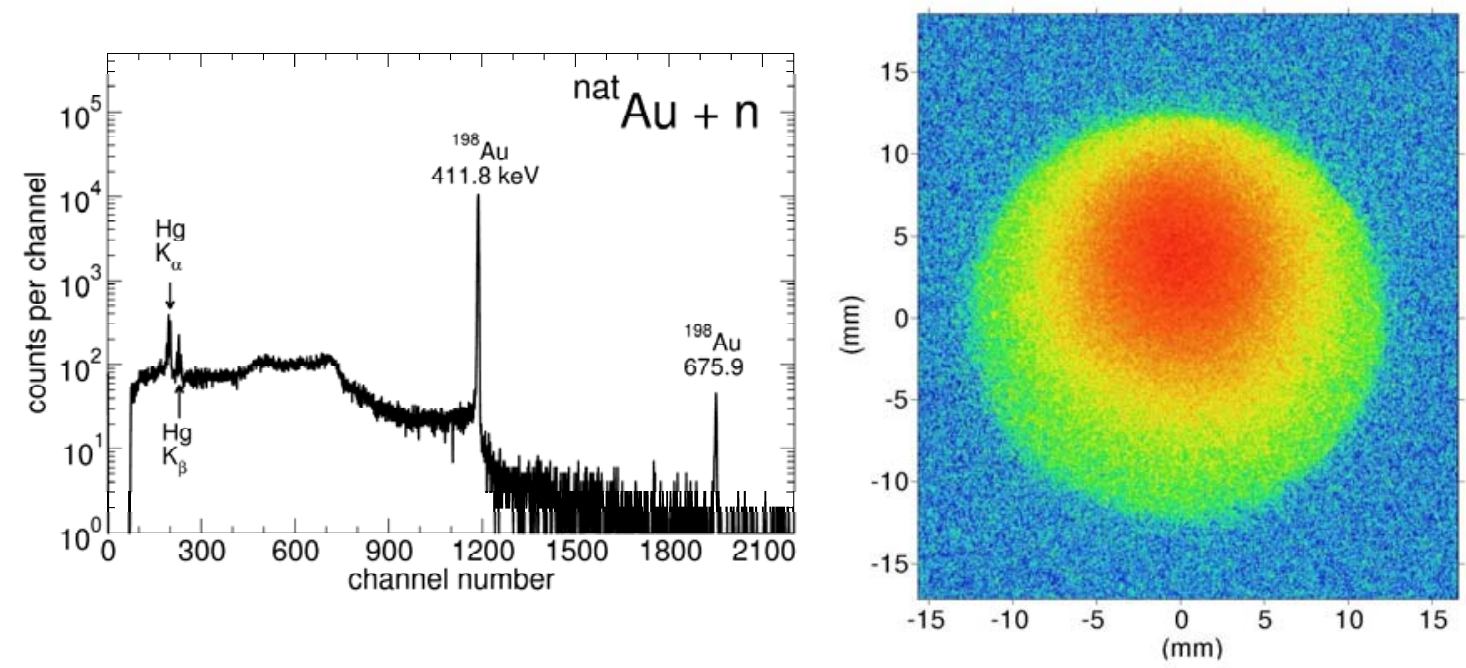

Figure 4: Gamma spectrum (left) and autoradiograph (right) of an Au monitor after activation in the LiLiT neutron flux showing the activity and activity distribution of the ${ }^{198} \mathrm{Au}$ nuclei produced by the ${ }^{197} \mathrm{Au}(n, \gamma)$ reaction. The autoradiograph is taken by placing the Au target on a photographic plate. The diameter of the Au target is $25 \mathrm{~mm}$ (green edge); a vertical offset of $\sim 3 \mathrm{~mm}$ from the center of the target is attributed to steering of the incident proton beam on the Li target. The offset is taken into account in the simulations.

collimated by the ${ }^{7} \operatorname{Li}(p, n)$ reaction kinematics, more than one order of magnitude larger than conventional neutron sources based on ${ }^{7} \mathrm{Li}(p, n)$. The energy distribution of neutrons intercepting the targets is illustrated in fig. 3 , as calculated by detailed simulations using the code SimLiT $[5,6]$ for neutron production from the ${ }^{7} \mathrm{Li}(p, n)$ reaction and GEANT4 [7] for the neutron transport to the activation targets. The codes were benchmarked by an experiment $[5,6]$ where angular and energy distributions were measured using a time-of-flight technique. Figure 4 illustrates a gamma spectrum and autoradiograph of a Au monitor target.

First activation experiments were performed with the SARAF-LiLiT setup with targets for which Maxwellian-averaged cross sections (MACS) are available in the literature. 
The ${ }^{\text {nat }} \mathrm{Zr}(60.8 \pm 0.5 \mathrm{mg})$ and ${ }^{\text {nat }} \mathrm{Ce}(374 \pm 0.5 \mathrm{mg})$ targets were selected and irradiated in two separate runs with proton charges of $1.1 \mathrm{~mA} . \mathrm{h}$ and $1.34 \mathrm{~mA} . \mathrm{h}$, respectively. The
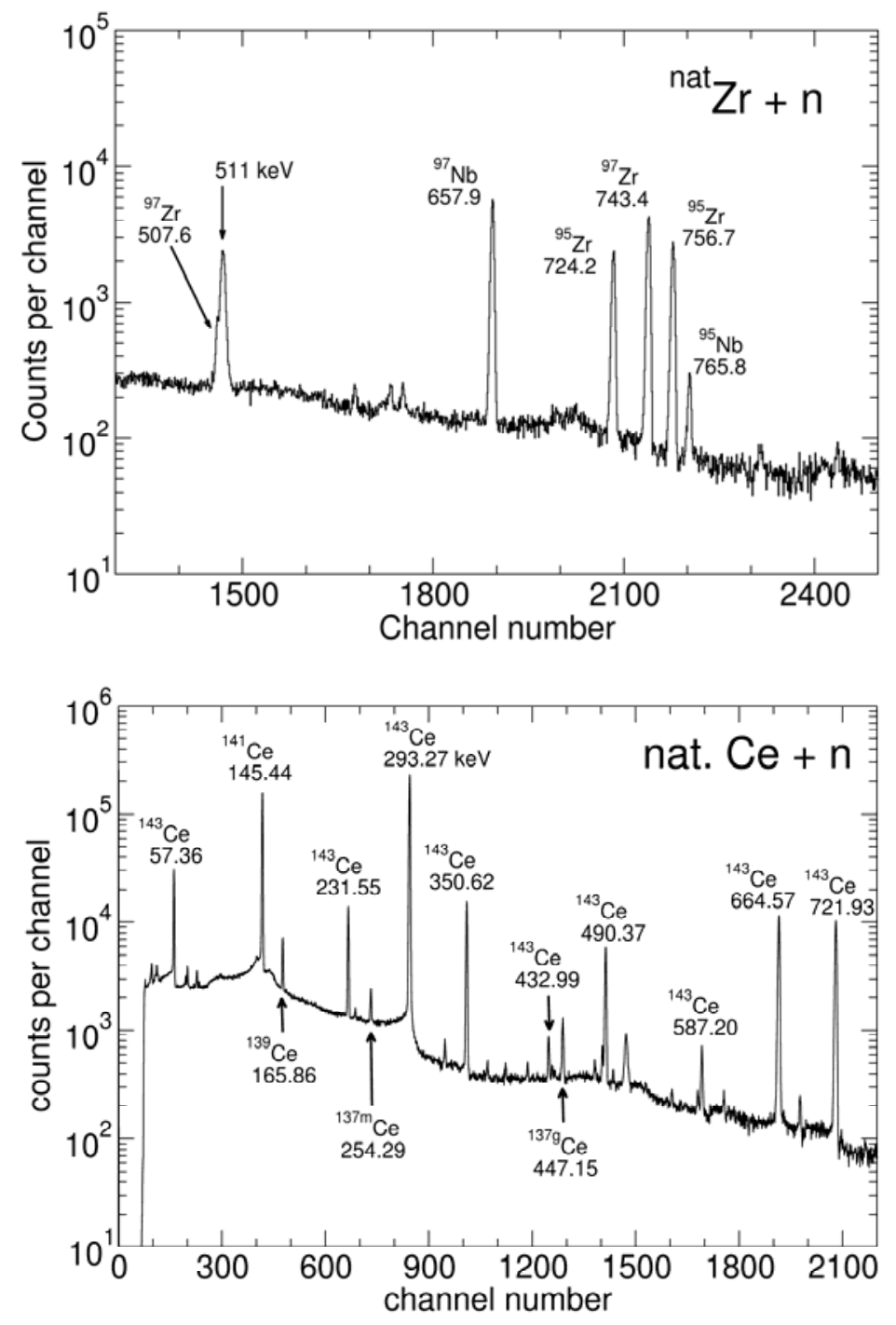

Figure 5: Gamma spectra accumulated with a shielded High-Purity Ge detector for targets of ${ }^{\text {nat }} \mathrm{Zr}$ and ${ }^{\text {nat }} \mathrm{Ce}$ (see text) activated in the neutron spectrum from LiLiT. The identified lines of the respective $(n, \gamma)$ reaction products are indicated.

proton beam energy and energy spread (typical to RF linear accelerators) were 1.91 $\mathrm{MeV}$ and $15 \mathrm{keV}$ respectively. Gamma spectra from the activated targets showing the major activation lines are displayed in fig. 5. Experimental cross sections (averaged over the neutron energy spectrum seen by the targets) are obtained from the ratio of activities of the investigated target to that of the Au monitors. Preliminary results for the 
MACS, extrapolated from these experimental cross sections using the ENDF/B-VII.1 library [8], are generally in good agreement with published values; table 1 lists preliminary results. The analysis takes into account systematic effects in the experiment such as proton beam energy and energy spread, target and target chamber geometry and materials; details of the methodology used for the extraction of cross sections and their uncertainties will be published separately. The case of the reaction ${ }^{138} \mathrm{Ce}(n, \gamma){ }^{139} \mathrm{Ce}$ where the target isotope has a very low natural abundance $(0.25 \%)$ and a residual nucleus with a half-life of 137.6 days stresses the importance of the high-intensity neutron source. Further analysis is in progress to reach final values of cross sections and uncertainties; the latter are expected to be reduced in the final analysis.

Table 1: Preliminary values of Maxwellian-averaged cross sections (MACS, mb) at $k \mathrm{~T}=30 \mathrm{keV}$ for stable isotopes of $\mathrm{Zr}$ and Ce measured with the high-intensity neutron source SARAF-LiLiT. The uncertainties quoted are conservative estimates, expected to be reduced in the final analysis.

\begin{tabular}{ccc}
\hline Nuclide & $\begin{array}{c}\text { Recommended, } \\
\text { KADoNiS [9] }\end{array}$ & $\begin{array}{c}\text { This work } \\
\text { (preliminary) }\end{array}$ \\
\hline${ }^{94} \mathrm{Zr}$ & $26 \pm 1$ & $29.0 \pm 1.5$ \\
${ }^{96} \mathrm{Zr}$ & $10.7 \pm 0.5$ & $13.1 \pm 0.9$ \\
${ }^{138} \mathrm{Ce}$ & $179 \pm 5$ & $302( \pm 10 \%)$ \\
${ }^{140} \mathrm{Ce}$ & $11.0 \pm 0.4$ & $10.5( \pm 10 \%)$ \\
${ }^{142} \mathrm{Ce}$ & $28 \pm 1$ & $30.4( \pm 10 \%)$ \\
\hline
\end{tabular}

\section{Conclusion}

The Liquid-Lithium Target LiLiT, operated with the high-intensity beam of the Soreq Applied Research Accelerator Facility SARAF, produces a quasi-Maxwellian flux of neutrons with an intensity larger by more than one order of magnitude than those of conventional setups used so far. First activation experiments performed with $\mathrm{Zr}$ and $\mathrm{Ce}$ targets of natural isotopic composition allow us to measure Maxwellian-averaged cross sections (MACS) and preliminary results show generally good agreement with published values. The experimental system is especially well suited for activation of low-abundance stable or radioactive targets by stellar-energy neutrons. 


\section{References}

[1] F. Kaeppeler, R. Gallino, S. Bisterzo, W. Aoki, Rev. Mod. Phys. 83 (2011) 157.

[2] S. Halfon et al., Rev. Sci. Instr. 84 (2013) 12350.

[3] S. Halfon et al., Rev. Sci. Instr. 85 (2014) 056105.

[4] A. Kreisel et al., in Proceedings of Linac 2014, Geneva (Switzerland), WEIOB02 (2014); and references therein..

[5] G. Feinberg et al., Phys. Rev. C 85 (2012) 055810.

[6] M. Friedman et al., Nucl. Instr. Meth. Phys. Res. A 698 (2013) 117.

[7] S. Agostinelli et al., Nucl. Inst. Meth. A 506 (3) (2003) 250.

[8] M.B. Chadwick et al., Nucl. Data Sheets 112 (2011) 2887.

[9] KADoNiS v0.3 - The third update of the "Karlsruhe Astrophysical Database of Nucleosynthesis in Stars", I. Dillmann et al., see http://www.kadonis.org/ 
